\title{
Optical Computing Systems, Materials, and Devices
}

\author{
Abdul A. S. Awwal \\ Wright State University \\ Computer Science and Engineering \\ Department \\ Dayton, Ohio 45435 \\ E-mail: aawwal@cs.wright.edu \\ Khan M. Iftekharuddin \\ University of Memphis \\ Department of Electrical and Computer \\ Engineering \\ 214 Engineering Science Building \\ Memphis, Tennessee 38152-3810 \\ E-mail: iftekhar@memphis.edu
}

The advancement in optical computing is impaired by a lack of development of optical computing devices leading to successful optical computing systems. In our special section published in March 1999, ${ }^{1}$ we aspired for more works in devices such that realistic optical systems might come to fruition. A similar concern was voiced recently, in the SPIE conference on Photonic Devices and Algorithms III held in San Diego. ${ }^{2,3}$ Therefore, this special section is a step toward addressing the needs in Optical Computing Systems, Materials, and Devices.

The papers in our special section are broadly organized into three groups: (1) analysis and simulation of optical computing devices and systems; (2) implementation of optical computing systems; and (3) optical device characterization and optical materials.

The largest cluster of papers appears in the area of analysis of optical computing devices and systems, such as hysteresis, memory capacity, bit-error in memory retrieval, speed, and noise. The paper by Marcos and Pereda discusses the influence of hysteresis in optical arithmetic units. The authors describe a novel technique to analyze the influence of possible hysteresis cycles in the devices employed for optical computing architectures. A simple full adder structure is used as the basis for the evaluation of the method. Nitta et al. perform string matching based on a spatial coded moiré technique for genome analysis. The string matching operation is achieved by overlapping of spatially coded patterns with a small intersection angle to generate moiré fringes. The moiré fringes give the matching information between two string sequences coded as the spatial patterns. Larichi analyzes a volume holographic memory system in order to find its bit error rate and system limitations. He proposes a model to estimate the capacity of volume holographic memories using system parameters such as bit error rate, data readout speed, and noise.

Systems simulation is an important part of systems design. The simulation of optical fuzzy logic systems and color-based pattern recognition systems is the subject of the next group of papers. One may argue that optics can be more effective somewhere in between strict binary and purely analog domain. Recent interest in fuzzy logic supports this assertion. Zhang and Karim propose a fuzzy logic processing system using a multi-object joint Fourier transform (JFT) processor. Their system calculates the firing strength using space-variant cross-correlation. The next paper in this group also deals with JFT for color image processing. Alam and Wai extend the concept of a fringe-adjusted filter to modify the power spectrum to achieve superior discrimination capability. Color channels are processed separately and then fused together to generate a single detection signal. In the final paper in this group, Caulfield proposes a new class of independent task filter analysis methodology that may be suitable for rapid parallel system implementation. The independent task Fourier filters may break complex computing tasks into space-variant manageable components.

The second group of papers addresses the optical computing systems implementation area. The proposed system implementations include VLSI optoelectronic circuits, VLSI-based SLMs, and electron-trapping material. Tang et al. discuss the design and analysis of single-chip optoelectronic database filters to address the requirements of next-generation archival storage systems. They have developed a single-chip database filter suitable for largecapacity database systems that use page-oriented optical storage devices. Based on a photonic VLSI device technology, their data filter monolithically integrates optical 
detectors, photoreceivers circuits, data manipulation logic, and filter control circuitry onto a single CMOS chip that can be readily fabricated using a standard VLSI fabrication facility. They also use queuing theory to show that even with the limitation of finite queue capacity, a database filter chip could be controlled to work at near optimal performance where database search time is limited by the data transfer rate going into the host computer.

Another recent trend captured in this issue is pushing optical interconnects into the traditional electronic circuits arena. The design of various interconnect systems using GRIN lenses and microlens arrays are described next. Baukens et al. discuss various trade-offs and design parameters for implementing free space optical interconnection systems for VLSI circuits. While a modified signed digit (MSD) number system leads to higher information density, if the number of steps is reduced, the truth tables may become too huge to be practical. Iftekharuddin et al. demonstrate the derivation of reduced minterms for a MSD adder circuit. The reduced minterms are implemented using a VLSI-based SLM truth table look-up process. The SLM serves as an encoder for the input to the optical computing system, while a fixed mask encodes the minimized truth table. While MSD allows for faster computation, one finally needs to convert the final stage output to a complement number system so that it can interact with other normal binary numbers. Li and Qian implement a converter for a signed-digit to complement number system using electron-trapping material. The electron trapping material allows for programmable logic operation.

Two authors address the area of optical materials and devices. Cohn reviews the properties of spatial light modulators, one of the most essential components of any optical computing system. The most current SLMs have a relatively low number of high-cost pixels with frequencydependent diffraction efficiency. Thus, the author's recommendation that encoding algorithms that address the entire usable frequency plane and emphasize fidelity of the approximated Fourier transform over maximization of diffraction efficiency is well taken. Grote et al. present the effects of various cladding material on nonlinear polymer based optoelectronic devices. They present the effect of using cladding materials with different conductivities and dielectric constants on the applied voltage for nonlinear optic (NLO) polymer based opto-electronic devices. Their findings suggest that only a small fraction of the applied modulation voltage reaches the NLO polymer core layer.

We hope this special section will generate enough interest such that progress is made toward realizing optical computing systems that can complement the more mature electronic counterparts. In the future, some of the interesting ideas presented in this special section may lead the researchers to new directions in this exciting field. We would like to thank all the authors for contributing to this special section, the reviewers for timely review of the papers selected here, and the Optical Engineering staff for their support and cooperation.

\section{References}

1. A. A. S. Awwal and K. M. Iftekharuddin, "Special Section Guest Editorial: Computer Arithmetic for Optical Computing," Opt. Eng. 38(3), 400-402 (1999).
2. F. T. S. Yu, "The legacy of optical information processing," Photonic Devices and Algorithm for Computing III, Proc. SPIE 4470 (2001).

3. N. Ooba and M. Okuno, "Planar-waveguide type optical switching devices," Photonic Devices and Algorithmis for Computing III, Proc. SPIE 4470 (2001).

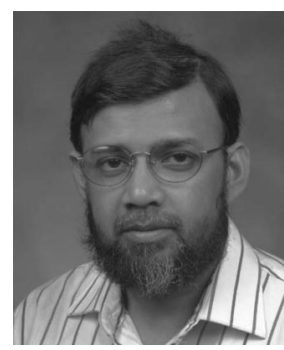

Abdul A. S. Awwal is an associate professor in the Computer Science and Engineering Department at Wright State University. He earned his PhD in electrical engineering from University of Dayton in 1989, his MS in electrical engineering from Whichita State University in 1986, and his BSc in electrical engineering with honors from Bangladesh University of Engineering and Technology (BUET) in 1984. He served as a lecturer in the Electrical Engineering department at BUET. Dr. Awwal received the "Excellence in Teaching" award in 1996 from the College of Engineering at WSU. Dr. Awwal's major research interests are in digital optical computing, digital systems design, computer arithmetic, multiple valued logic, neural networks, pattern recognition, display devices, electro-optical sensors, and digital/optical image/signal processing. He co-authored a graduate level textbook entitled $\mathrm{Op}$ tical Computing: An Introduction published by John Wiley in 1992. In addition, Dr. Awwal has published more than 60 refereed journal papers, two book chapters, and more than 80 conference publications. He is a member of IEEE, American Society for Engineering Education, Optical Society of America, and the International Society for Optical Engineering (SPIE). He has served as a reviewer for IEEE Computer, National Science Foundation, Optical Engineering, Optics Letters, Journal of Optical Society of America, Applied Optics, Optics Communications, and Optics and Laser Technology.

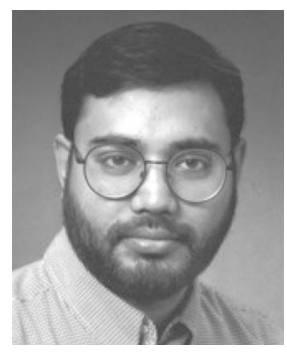

Khan M. Iftekharuddin is an assistant professor in the Department of Electrical and Computer Engineering at the University of Memphis (U of M). Prior to the $U$ of $M$, he was on the faculty of the Departments of Computer Science and Electrical \& Computer Engineering at North Dakota State University (NDSU) for two years. He obtained his BSc degree from Bangladesh Institute of Technology in 1989. He received an MS and a PhD both in electrical engineering from the University of Dayton in 1991 and 1995, respectively. His research interests include optical computing and interconnection, sensor signal acquisition and modeling, signal and image processing, embedded systems, biologically inspired systems, applications of neuro-fuzzy inference techniques, and automatic target recognition (ATR). Dr. Iftekharuddin was a principal research engineer at Timken Research, Canton, Ohio, before joining NDSU. There he was involved in research in signal and image processing, neural networks applications, timefrequency analysis, sensors, embedded system design, and optical interconnection networks. Much of his research had focused on ATR problems. Prior to Timken, Dr. Iftekharuddin was a senior systems engineer at BDM International in Dayton, Ohio. There he was involved in software development, software process improvement, and ATR research. He was named the researcher of the year in the College of Engineering and Architecture at NDSU for 2000. He is the principal author of more than 50 refereed journal papers and conference proceedings and a book chapter on optical interconnection networks published in Advances in Electronics and Electron Physics (Academic Press, 1997). He was a co-editor for two special sections on Optical Computing Algorithms, Devices, and Materials in Optical Engineering in March 1999 and November 2001, respectively. He was also a co-editor of a special issue on Optical Computing Arithmetic and Systems in Optics and Laser Technology in September 2000. He has been a lead conference chairman and organizer of an annual conference on Photonic Devices and Algorithms for Computing at SPIE's International Symposium on Optical Science, Engineering, and Instrumentation since 1999 . He is a member of IEEE, OSA, ASEE, and SPIE. 